\title{
Exact Solutions for Certain Nonlinear Autonomous Ordinary Differential Equations of the Second Order and Families of Two-Dimensional Autonomous Systems
}

\author{
M. P. Markakis \\ Department of Engineering Sciences, University of Patras, 26504 Patras, Greece \\ Correspondence should be addressed to M. P. Markakis, m.markakis@des.upatras.gr \\ Received 19 August 2010; Revised 22 November 2010; Accepted 30 November 2010 \\ Academic Editor: Peiguang Wang \\ Copyright (C) 2010 M. P. Markakis. This is an open access article distributed under the Creative \\ Commons Attribution License, which permits unrestricted use, distribution, and reproduction in \\ any medium, provided the original work is properly cited. \\ Certain nonlinear autonomous ordinary differential equations of the second order are reduced \\ to Abel equations of the first kind ((Ab-1) equations). Based on the results of a previous work, \\ concerning a closed-form solution of a general (Ab-1) equation, and introducing an arbitrary \\ function, exact one-parameter families of solutions are derived for the original autonomous \\ equations, for the most of which only first integrals (in closed or parametric form) have been \\ obtained so far. Two-dimensional autonomous systems of differential equations of the first order, \\ equivalent to the considered herein autonomous forms, are constructed and solved by means of \\ the developed analysis.
}

\section{Introduction}

Autonomous equations, as it is well known, often arise in mechanics, physics, and chemical engineering since a considerable number of problems are governed by weakly or strongly nonlinear equations of this kind. For example in the study of damped oscillators one records famous equations, extensively investigated in the literature, like the ones governing the Duffing [1] or the Van der Pol [2] oscillator. Both these equations are of the Liénard type:

$$
\frac{d^{2} y}{d x^{2}}+k(y) \frac{d y}{d x}+m(y)=0
$$

where $k$ and $m$ are differentiable functions of $y$. Furthermore, (1.1) as well as other autonomous equations like the Rayleigh [3] or the generalized mixed Rayleigh-Liénard 
equation [4], are special cases of the more general form

$$
\frac{d^{2} y}{d x^{2}}+[k(y)+l(q)] \frac{d y}{d x}+m(y)=0, \quad q=\frac{d y}{d x}
$$

with $l$ a differentiable function of $q$. Equations (1.1) and (1.2) have been studied thoroughly in the literature with regard to the stability of their critical points and the number of the limit cycles which correspond to global or local bifurcations, especially in the case where $k, l, m$ are polynomials. For example, we refer to [5-9] as far as (1.1) is concerned and $[4,10,11]$ for the more generalized case (1.2). We should also note that a lot of authors obtain exact or approximate first integrals (also called adiabatic invariants). See for instance the work of Kooij and Christopher for the integrability of planar polynomial systems by means of algebraic invariant curves [12], as well as the works of Denman [13] and Van Horssen [14], where approximate invariants are obtained via perturbation techniques. Moreover, numerous works based on various perturbation methods yield approximate solutions and (or) qualitative results (see the book of Verhulst [15] and the references there in). In addition, some series solutions have been derived in the literature, concerning nonlinear ODEs, where the developed methods can be applied in general to cases of autonomous equations. For example, we refer to functional analytic techniques resulting in this kind of solutions (see $[16,17])$. However, not much progress has been made as regards the derivation of exact, general, closed-form solutions of the equations studied in the above references. Thus by considering autonomous equations of a polynomial structure for $d y / d x$ (up to the second degree), with coefficients of a not necessarily polynomial form for $y$ (see, e.g., the Langmuir equation [18]), in the present work we investigate analytically this generalized polynomial form, aiming at the construction of proper techniques, capable of removing the difficulties arising in the derivation of exact solutions. (Most of the above mentioned equations are presented by Davis [19, Chapter 7, Section 2].)

A significant part of the relevant search in procedures of this kind deals with the use of appropriate transformations. However, the classic transformation $y_{x}^{\prime}=q(y)$, usually applied to autonomous nonlinear ordinary differential equations of the second order results in Abel equations of the second kind (see, e.g., [20, Section 2.2.3]), which in general cannot be solved analytically, except in special cases, most of which accept only parametric solutions (see [20, Sections 1.3.1-1.3.4]) (therefore a parametric solution for $y, y_{x}^{\prime}$ is derived as regards the considered autonomous equation). Hence, in Section 2, in order to construct a more efficient analytical technique, concerning two general subclasses of the autonomous equations under consideration, we use another, properly modified, general transformation, to obtain Abel equations of the first kind. Furthermore in [21], an implicit solution of a general (Ab-1) equation has been obtained, together with the associated sufficient condition.

Then in Section 3, introduction of an arbitrary function in combination with the derived (in [21]) solution, yield one-parameter families of solutions for the original nonlinear autonomous equation. More specifically, by means of the sufficient condition being extracted for the solution of the Abel equation, the arbitrary function is determined so that a first integral (of the autonomous equation) of the form $d y / d x=h(y)$ is to be derived. As application, we obtain families of solutions concerning four specific cases of Liénard equations, for which first integrals in parametric form (or parametric solutions) have been derived so far. Finally, in Section 4, by using the solutions extracted in Section 3, we conclude solutions for families of nonlinear autonomous systems of differential equations of the first order, equivalent to the equations considered in this work. Two examples of such families 
are given, where solutions are obtained in combination with solvable cases of autonomous equations, presented in Section 3.

\section{Reduction of a General Autonomous Equation}

Hereafter the prime denotes differentiation with respect to the corresponding suffix. We consider the following general autonomous differential equation of the second order:

$$
y_{x x}^{\prime \prime}+\sum_{i=1}^{n} g_{i}(y)\left(y_{x}^{\prime}\right)^{i}+g_{0}(y)=0, \quad g_{0} \ldots g_{n} \neq 0, y=y(x), n=1,2
$$

with $g_{i}(y), i=0, \ldots, n$, continuous functions of $y$. In particular for $n=1$ we have the Liénard equation. By applying the transformation $(M)$ :

$$
(M): y_{x}^{\prime}=\frac{1}{p(y)}
$$

we arrive at the $(\mathrm{Ab}-1)$ equation:

$$
\begin{gathered}
n=1: p_{y}^{\prime}=g_{0} p^{3}+g_{1} p^{2}, \\
n=2: p_{y}^{\prime}=g_{0} p^{3}+g_{1} p^{2}+g_{2} p .
\end{gathered}
$$

We further consider the Abel equation of the first kind:

$$
y_{x}^{\prime}=f_{3}(x) y^{3}+f_{2}(x) y^{2}+f_{1}(x) y, \quad y=y(x)
$$

Then in [21], by taking into account a solvable in closed-form Abel equation [20, Section 1.4.1.47 $(c=-a / b)]$, involving arbitrary functions and using a transformation given by Kamke [22, Chapter A, Equation 4.10.d], we have finally proved the following theorem.

Theorem 2.1. If the following relation holds:

$$
\left(\frac{f_{3}}{f_{2}}\right)_{x}^{\prime}=\frac{c}{(1+c)^{2}} f_{2}-\frac{f_{3} f_{1}}{f_{2}}, \quad c \neq \pm 1
$$

then the Abel equation (2.4) $\left(f_{2} \neq 0\right)$ has a general implicit solution of the form

$$
\left|1+\frac{f_{2}(x)}{(1+c) y(x) f_{3}(x)}\right|^{c}\left|1+\frac{c f_{2}(x)}{(1+c) y(x) f_{3}(x)}\right|^{-1}-K \exp \left[\frac{c(1-c)}{(1+c)^{2}} \int \frac{f_{2}{ }^{2}}{f_{3}} d x\right]=0,
$$

where $c$ is an arbitrary parameter and $K$ stands for the parameter of the family of solutions.

In fact, one more sufficient condition is extracted together with (2.5) (see [21, Equation (3.2)]), constituting a relation between the arbitrary functions involved in the auxiliary 
"arbitrary" equation. These functions are not included in the extracted solution and hence we can claim that they are finally eliminated, "allowing" the derivation of the closed-form solution (2.6). We also note that a similar condition is given by Kamke [22, Chapter A, Equation 4.10.f], limited to the Liénard equations, but the resulting implicit formulas have a rather more complicated structure than the equation obtained here as regards the "algebraic" evaluation of the dependent variable (see [21, Equations (3.16)-(3.18)]).

\section{Construction of Exact Solutions}

Let us consider a first order ordinary differential equation of the general form:

$$
y_{x}^{\prime}=F(x, y), \quad y=y(x)
$$

By introducing an arbitrary function $G(x)$, we write the system of equations:

$$
\begin{array}{r}
y_{x}^{\prime}=-F_{1}(x, y)+G(x) f(y), \\
y_{x}^{\prime}=\frac{1}{2} F_{2}(x, y)+\frac{1}{2} G(x) f(y), \\
F(x, y)=F_{1}(x, y)+F_{2}(x, y),
\end{array}
$$

where $f$ is a known continuous function of $y$.

Proposition 3.1. If there exists a function $G(x)$ such that (3.2) and (3.3) have a common solution, then this solution satisfies (3.1) as well.

Proof. Proposition 3.1 follows easily, since by combining (3.2) and (3.3) and taking into account (3.4), we obtain (3.1).

By replacing now $x$ with $y$ and $y(x)$ with $p(y)$, we write (3.2) and (3.3) with respect to $(2.3 \mathrm{~b})$ (or $(2.3 \mathrm{a})$ when $\left.n=1\left(g_{2}=0\right)\right)$, as

$$
\begin{gathered}
p_{y}^{\prime}=\left[G(y)-g_{0}\right] p^{3}-g_{1} p^{2}-g_{2} p, \\
p_{y}^{\prime}=\frac{G(y)}{2} p^{3},
\end{gathered}
$$

with $F_{1}(y, p)=g_{0} p^{3}+g_{1} p^{2}+g_{2} p, F_{2}(y, p)=0$, and $f(p)=p^{3}$. Then, by means of Proposition 3.1, we prove the following theorem.

Theorem 3.2. The nonlinear autonomous equation (2.1) has the following exact one-parameter families of solutions:

$$
\int \frac{d y}{\sqrt{B-\int G(y) d y}}= \pm x+A
$$


where A stands for the parameter of the family, $G$ is given by

$$
G(y)=g_{1} \exp \left(\int g_{2} d y\right)\left[\Gamma+\kappa \int g_{1} \exp \left(-\int g_{2} d y\right) d y\right]+g_{0}
$$

and the parameters $\kappa, \Gamma, B$ are evaluated by means of the following relation:

$$
\frac{G-2 g_{0}}{g_{1}}= \pm 2 \sqrt{B-\int G d y}+\frac{2 g_{2}}{g_{1}}\left(B-\int G d y\right)
$$

with $G$ as in (3.8).

Proof. Since (3.5) is an (Ab-1) equation of the form (2.4) (with $y$ instead of $x$ and $p(y)$ instead of $y(x)$ ), application of the sufficient condition (2.5) (where $x$ is replaced by $y$ and $f_{3}(y)=$ $\left.G-g_{0}, f_{2}(y)=-g_{1}, f_{1}(y)=-g_{2}\right)$ results in the linear equation:

$$
\left(\frac{G-g_{0}}{g_{1}}\right)_{y}^{\prime}=g_{2} \frac{G-g_{0}}{g_{1}}+\kappa g_{1}, \quad \kappa=\frac{c}{(1+c)^{2}}
$$

Then (3.8) is obtained as the solution of (3.10). Moreover, according to Theorem 2.1, (3.5) has a closed-form solution, given by (2.6) (modified as regards the variables). On the other hand, integration of the separated variables equation (3.6) yields

$$
y_{x}^{\prime}=\frac{1}{p(y)}= \pm \sqrt{\mathrm{B}-\int G(y) d y}
$$

where $\mathrm{B}$ is an integration constant.

By equating now the right-hand sides of (3.5) and (3.6), we conclude to the relation:

$$
G=2 g_{0}+\frac{2 g_{1}}{p}+\frac{2 g_{2}}{p^{2}}
$$

where substitution of (3.11) for $p$, results in (3.9). The parameters $\kappa, \Gamma$ involved in the expression obtained for $G$ (3.8), as well as the parameter B appearing in (3.11), can be determined by means of (3.9), where (3.8) is substituted for $G$. Hence, the function $G(y)$ can be determined so that (3.5) and (3.6) to have a common solution given from (3.11) (or (2.6)). It follows from Proposition 3.1 that this solution satisfies (2.3b) (or (2.3a) when $n=1$ ) and therefore constitutes a first integral of the autonomous (2.1). Finally integration of (3.11) yields (3.7) and the proof of the theorem is complete.

Thus use of Theorem 3.2 in the case of an autonomous equation of the form (2.1), means that we determine at first the parameters $\kappa, \Gamma$, B by means of (3.9), then we obtain $G(y)$ by using (3.8), and finally by substituting B and $G$ in (3.7) we arrive at a one-parameter family of solutions for the considered equation. We apply now this procedure to four cases of Liénard equations. The first case (Example 3.3) concerns the general form of an equation the 
solution of which can not be found in [20]. In fact, a special case of this equation is presented by Polyanin and Zaitsev, where the proposed procedure results in a parametric solution for $y, y_{x}^{\prime}$. The same authors [20, Section 2.2.3] arrive at this kind of solution for the other two cases as well (Examples 3.4 and 3.6) (by means of the classic transformation $y_{x}^{\prime}=q(y)$, the equations are finally reduced to Abel forms of the second kind), while as regards the fourth case (Example 3.5), in [20] a parametric solution for $x, y$ is presented. In general, as regards the parametric solutions, besides the parameter can not be eliminated (except for very special cases (in this case a first integral would be derived for the considered autonomous forms)), it should be noted that this kind of solutions cannot be handled easily, since in many cases it is very difficult to determine the domain of validity of the parameter for the problem under consideration. Moreover, it is worth to be mentioned that any of the reduced (Ab-1) equations (2.3a) and (2.3b), which correspond to the considered examples, is included in the solvable cases of the Abel equations of the first kind presented in [20, Section 1.4.1].

Example 3.3. We consider the equation

$$
y_{x x}^{\prime \prime}+a y^{2 n-1} y_{x}^{\prime}+\beta y^{2 n-1}+\gamma y^{4 n-1}=0
$$

with $g_{2}(y)=0, g_{1}(y)=a y^{2 n-1}, g_{0}(y)=\beta y^{2 n-1}+r y^{4 n-1}$. A special case of (3.13) is presented in [20, Section 2.2.3.8 $(b=0, n=k)]$, that is,

$$
y_{x x}^{\prime \prime}-3 a n y^{2 n-1} y_{x}^{\prime}-c y^{2 n-1}-a^{2} n y^{4 n-1}=0,
$$

where the transformation $y_{x}^{\prime}=p(y)=y^{n}\left(\tau+a y^{n}\right)$ yields a Bernoulli equation with respect to $y(\tau)$, and finally a parametric solution is extracted, namely,

$$
y=\phi(\tau ; \mathrm{A}), \quad y_{x}^{\prime}=\phi^{n}(\tau ; \mathrm{A})\left[\tau+a \phi^{n}(\tau ; \mathrm{A})\right],
$$

with A an integration constant. For the general case (3.13) Theorem 3.2 implies

$$
\begin{gathered}
G(y)=(a \Gamma+\beta) y^{2 n-1}+\left(\kappa \frac{a^{2}}{2 n}+\gamma\right) y^{4 n-1}, \\
(\kappa, \Gamma, \mathrm{B})=\left(-1+\frac{2 \gamma n}{a^{2}} \pm \sqrt{1-\frac{8 \gamma n}{a^{2}}}, \frac{a \beta\left(\kappa-2-2 \gamma n / a^{2}\right)}{a^{2}(\kappa+2)-2 \gamma n}, \frac{4 a^{2} \beta^{2}}{\left[a^{2}(\kappa+2)-2 \gamma n\right]^{2}}\right) .
\end{gathered}
$$

Thus (3.7) takes the form

$$
\int \frac{d y}{\sqrt{\mathrm{B}-(1 / 2 n)(a \Gamma+\beta) y^{2 n}+1 / 4 n\left(\kappa\left(a^{2} / 2 n\right)+\gamma\right) y^{4 n}}}= \pm x+\mathrm{A} .
$$


For $n=1$, (3.13) becomes

$$
y_{x x}^{\prime \prime}+a y y_{x}^{\prime}+\beta y+\gamma y^{3}=0
$$

for which in [20, Equation 2.2.3.2] a parametric solution is also obtained. Here, considering for example the set of parameters:

$$
(a, \beta, \gamma)=(3,1,1)
$$

by (3.18) $(n=1)$ two one-parameter families of solutions for (3.19) are derived:

$$
\begin{aligned}
& \left(\kappa=-\frac{4}{9}, \Gamma=-\frac{2}{3}, \mathrm{~B}=\frac{1}{4}\right) \Longrightarrow y=\tan \left(\frac{-x+\mathrm{A}}{2}\right), \\
& \left(\kappa=-\frac{10}{9}, \Gamma=-\frac{5}{3}, \mathrm{~B}=1\right) \Longrightarrow y=\tan (-x+\mathrm{A}) .
\end{aligned}
$$

Example 3.4. Using the equation in $[20,2.2 .3 .7(b=0)]$,

$$
y_{x x}^{\prime \prime}+a y^{n} y_{x}^{\prime}+r y^{2 n+1}=0
$$

with $g_{2}(y)=0, g_{1}(y)=a y^{n}, g_{0}(y)=r y^{2 n+1}$, we obtain

$$
\begin{gathered}
G(y)=a \Gamma y^{n}+\left(\gamma+\kappa \frac{a^{2}}{n+1}\right) y^{2 n+1}, \\
(k, \Gamma, \mathrm{B})=\left(-1+\frac{\gamma(n+1)}{a^{2}} \pm \sqrt{1-\frac{4 \gamma(n+1)}{a^{2}}}, 0,0\right),
\end{gathered}
$$

Then (3.7) becomes

$$
-\frac{1}{n}\left[-\frac{\kappa a^{2}+\gamma(n+1)}{2(n+1)^{2}}\right]^{-1 / 2} y^{-n}= \pm x+\mathrm{A}
$$

For $(n, a, \gamma)=(1,-3,1),(3.24)$ results in the following solutions:

$$
\kappa=-\frac{10}{9} \Longrightarrow y=\frac{1}{-x+\mathrm{A}}, \quad \kappa=-\frac{4}{9} \Longrightarrow y=\frac{2}{-x+\mathrm{A}},
$$

while for $(n, a, \gamma)=(1 / 2,-2,1 / 2)$ we have that

$$
\kappa=-\frac{21}{16} \Longrightarrow y=\frac{4}{(x+\mathrm{A})^{2}}, \quad \kappa=-\frac{5}{16} \Longrightarrow y=\frac{36}{(x+\mathrm{A})^{2}} .
$$


Example 3.5. We study the equation

$$
y_{x x}^{\prime \prime}+a f(y) y_{x}^{\prime}+b f(y)=0
$$

with $g_{2}(y)=0, g_{1}(y)=a f(y), g_{0}(y)=b f(y)$, and $f$ any continuous function of $y$. In [20] three equations of the form (3.27) are presented [20, Section 2.2.3.10 $\left(f=e^{\lambda y}\right)$, Section 2.2.3.19 $(f=\sin (\lambda y))$, Section 2.2.3.20 $(f=\cos (\lambda y))$ ]. Here we have that

$$
\begin{gathered}
G(y)=(a \Gamma+b) f+\kappa a^{2} f \int f d y, \\
(\kappa, \Gamma, \mathrm{B})=\left(0,-\frac{b}{a}, \frac{b^{2}}{a^{2}}\right) .
\end{gathered}
$$

Thus (3.7) concludes to the solution

$$
y=-\frac{b}{a} x+\mathrm{A}
$$

Example 3.6. Using the equation in [20, Section 2.2.3.11 $(b=0)]$,

$$
y_{x x}^{\prime \prime}+a e^{y} y_{x}^{\prime}-\gamma e^{2 y}=0
$$

with $g_{2}(y)=0, g_{1}(y)=a e^{y}, g_{0}(y)=-\gamma e^{2 y}$, relations (3.8) and (3.9) yield

$$
\begin{gathered}
G(y)=a \Gamma e^{y}+\left(\kappa a^{2}-\gamma\right) e^{2 y} \\
(\kappa, \Gamma, \mathrm{B})=\left(-1-\frac{\gamma}{a^{2}} \pm \sqrt{1+\frac{4 \gamma}{a^{2}}}, 0,0\right)
\end{gathered}
$$

Then by (3.7) we derive

$$
\begin{aligned}
& y=\ln \left(\sqrt{\frac{2}{\gamma-\kappa a^{2}}} \frac{1}{-x+\mathrm{A}}\right), \quad a\left(\gamma+\kappa a^{2}\right)>0, \\
& y=\ln \left(\sqrt{\frac{2}{\gamma-\kappa a^{2}}} \frac{1}{x+\mathrm{A}}\right), \quad a\left(\gamma+\kappa a^{2}\right)<0 .
\end{aligned}
$$

where $\kappa$ is given by (3.32).

We should note that in certain cases, depending on the form of $g_{i}(y), i=0,1,2$, in order to determine $\kappa, \Gamma$, B by means of (3.9), we may need to determine one or more parameters of the original equation, as well, or establish appropriate relations concerning these parameters. This means that application of Theorem 3.2 yields families of solutions valid for special cases 
of the considered equation. Obviously, when these special cases concern "degenerate" forms, like the linearized ones or equations with $g_{0}=0$, then the developed herein analytical method becomes not appropriate for the specific autonomous equation. For example, if we consider an equation of the form

$$
y_{x x}^{\prime \prime}+\left(a_{0}+a y^{2}\right) y_{x}^{\prime}+\beta_{0}+\beta y+r y^{3}=0, \quad a \neq 0,
$$

which is the general form of the reduced second-order equation corresponding to the Fitzhugh-Nagumo system (with $x(t)$ instead of $y(x)$, see [17]), then by (3.9) we obtain $(\kappa, \Gamma, \mathrm{B})=(0,0,0)$, holding for the case where $\left(\beta_{0}, \beta, \gamma\right)=(0,0,0)$. On the other hand, an example where the present analysis arrives at exact solutions for special cases of the considered equation, is the following:

$$
y_{x x}^{\prime \prime}+\left(a_{0}+a y^{2}\right) y_{x}^{\prime}+\beta_{0}+\beta y^{2}=0, \quad a \neq 0
$$

Here, (3.9) results in $(\kappa, \Gamma, \mathrm{B})=\left(0,-\left(\beta_{0} / a_{0}\right),\left(\beta_{0}^{2} / a_{0}^{2}\right)\right)$, valid for the case where $\left(a / a_{0}\right)=$ $\left(\beta / \beta_{0}\right)=\varepsilon$, which is the case of (3.27) with $(a, b)=\left(a_{0}, \beta_{0}\right)$ and $f(y)=1+\varepsilon y^{2}$, accepting the solution $y=-\left(\beta_{0} / a_{0}\right) x+\mathrm{A}$.

\section{Autonomous Systems Equivalent to (2.1) and Exact Solutions}

By constructing two-dimensional autonomous systems of differential equations equivalent to (2.1), then based on Theorem 3.2 we can prove the following proposition.

Proposition 4.1. The autonomous system of ordinary differential equations of the first order:

$$
\begin{gathered}
x_{t}^{\prime}=-\frac{1}{g_{, x}}\left\{g_{2}(y) g^{2}(x, y)+\left[g_{, y}+g_{1}(y)\right] g(x, y)+g_{0}(y)\right\}, \quad x=(x) t, \\
y_{x}^{\prime}=g(x, y), \quad y=y(t),
\end{gathered}
$$

where $g(x, y)$ is an arbitrary function with continuous partial derivatives $g_{, x}, g_{y}$, and $g_{i}(y), i=$ $0,1,2, g_{0} g_{1} \neq 0$, continuous functions of $y$, has the following one-parameter family of solutions:

$$
\int \frac{d y}{\sqrt{B-\int G(y) d y}}= \pm t+A, \quad \pm \sqrt{B-\int G(y) d y}=g(x, y)
$$

where A represents the parameter of the family, $G(y)$ is given by (3.8) and the parameters $\kappa, \Gamma, B$ are obtained by (3.9).

Proof. Let us consider the following system:

$$
\begin{array}{ll}
x_{t}^{\prime}=h(x, y), & x=(x) t, \\
y_{t}^{\prime}=g(x, y), & y=y(t),
\end{array}
$$


with $g$ arbitrary function with continuous partial derivatives of the first order and $h$ another arbitrary function. We additionally consider $(2.1)(n=2)$ where $x$ is replaced by $t$, namely,

$$
y_{t t}^{\prime \prime}+g_{2}(y) y_{t}^{\prime 2}+g_{1}(y) y_{t}^{\prime}+g_{0}(y)=0, \quad g_{0} g_{1} \neq 0, y=y(t)
$$

By differentiating now the second equation of (4.3) with respect to $t$ and substituting relations (4.3) for the derivatives, we obtain

$$
y_{t t}^{\prime \prime}=g_{, x} h(x, y)+g_{, y} g(x, y)
$$

Thus by substituting the right-hand sides of the second equation of (4.3) and (4.5), for $y_{t}^{\prime}$ and $y_{t t}^{\prime \prime}$, respectively, and solving for $h\left(=x_{t}^{\prime}\right),(4.4)$ concludes to the first of (4.1). Therefore the system (4.1) is equivalent to (4.4) and hence, from Theorem 3.2 it follows that the solution for $y(t)$ is given by the first equation of (4.2), while combination of (3.11) (with $t$ instead of $x$ ) with the second equation of (4.1) yields the second equation of (4.2). The proof of the proposition is complete.

The aim of the above "constructive" proposition is that, for every function $g(x, y)$ possessing continuous partial derivatives, a family of nonlinear autonomous systems can be constructed by means of (4.1), which can be solved exactly via (3.8), (3.9), and (4.2). For example, we consider two specific forms for $g$, combined with solved autonomous equations, presented in Section 3.

(1)

$$
g(x, y)=\mathrm{A}_{0}+A x+B y+L x^{2}+M x y+N y^{2}
$$

that is the general quadratic case.

(a) Regarding (3.19), we write the equivalent nonlinear system (4.1), namely,

$$
\begin{gathered}
x_{t}^{\prime}=-\frac{1}{\mathrm{~A}+2 L x+M y}\left(C_{00}+C_{10} x+C_{01} y+C_{20} x^{2}+C_{11} x y+C_{02} y^{2}\right. \\
\left.+C_{30} x^{3}+C_{21} x^{2} y+C_{12} x y^{2}+C_{03} y^{3}\right) \\
y_{t}^{\prime}=\mathrm{A}_{0}+A x+B y+L x^{2}+M x y+N y^{2},
\end{gathered}
$$

where $C_{i j}=C_{i j}\left(\mathrm{~A}_{0}, \mathrm{~A}, \mathrm{~B}, L, M, N, a, \beta, \gamma\right), i, j=0, \ldots, 3$. The specific expressions of the coefficients $C_{i j}$ can easily be obtained by the first equation of (4.1) and hence it is not necessary to be given here. Taking now into account the results obtained above (Section 3, Example 3.3), for the set of parameters $(a, \beta, \gamma)=(3,1,1),(4.2)$ 
yield

$$
\begin{aligned}
&\left(\kappa=-\frac{4}{9}, \Gamma=-\frac{2}{3}, \mathrm{~B}=\frac{1}{4}\right) \Longrightarrow y=\tan \left(\frac{-t+\mathrm{A}}{2}\right), \\
& \mathrm{A}_{0}+\frac{1}{2}+A x+B y+L x^{2}+M x y+\left(N+\frac{1}{2}\right) y^{2}=0, \\
&\left(\kappa=-\frac{10}{9}, \quad \Gamma=-\frac{5}{3}, \quad \mathrm{~B}=1\right) \Longrightarrow y=\tan (-t+\mathrm{A}), \\
& \mathrm{A}_{0}+1+A x+B y+L x^{2}+M x y+(N+1) y^{2}=0 .
\end{aligned}
$$

(b) As a second example of an autonomous system based on (4.6), we consider (3.27), where the associated system (4.1) takes the form

$$
\begin{aligned}
& x_{t}^{\prime}=-\frac{1}{\mathrm{~A}+2 L x+M y}\{ D_{00}+D_{10} x+D_{01} y+D_{20} x^{2}+D_{11} x y+D_{02} y^{2} \\
&+D_{30} x^{3}+D_{21} x^{2} y+D_{12} x y^{2}+D_{03} y^{3} \\
&+ {\left.\left[a\left(\mathrm{~A}_{0}+A x+B y+L x^{2}+M x y+N y^{2}\right)+b\right] f(y)\right\} } \\
& y_{t}^{\prime}=\mathrm{A}_{0}+A x+B y+L x^{2}+M x y+N y^{2}
\end{aligned}
$$

where the coefficients $D_{i j}=D_{i j}\left(\mathrm{~A}_{0}, \mathrm{~A}, \mathrm{~B}, L, M, N\right), i, j=0, \ldots, 3$ are obtained by the first equation of (4.1). By means of the solution extracted above (Section 3, Example 3.5), (4.2) result in

$$
\begin{gathered}
y=-\frac{b}{a} t+\mathrm{A} \\
\mathrm{A}_{0}+\frac{b}{a}+A x+B y+L x^{2}+M x y+N y^{2}=0 .
\end{gathered}
$$

(2)

$$
g(x, y)=\left(\mathrm{A}_{0}+A x+B y\right) e^{y}
$$

Here, considering (3.30), by (4.1) we form the equivalent system

$$
\begin{gathered}
x_{t}^{\prime}=-\frac{1}{\mathrm{~A}}\left(E_{00}+E_{10} x+E_{01} y+E_{20} x^{2}+E_{11} x y+E_{02} y^{2}\right) e^{y}, \\
y_{t}^{\prime}=\left(\mathrm{A}_{0}+A x+B y\right) e^{y},
\end{gathered}
$$


with $E_{i j}=E_{i j}\left(\mathrm{~A}_{0}, \mathrm{~A}, \mathrm{~B}, a, \gamma\right), i, j=0, \ldots, 3$, provided from the first of (4.1). According to the derived solutions in the Example 3.6 of Section 3, (4.2) conclude to

$$
\begin{array}{ll}
a\left(\gamma+\kappa a^{2}\right)>0 \Longrightarrow y=\ln \left(\sqrt{\frac{2}{r-\kappa a^{2}}} \frac{1}{-t+\mathrm{A}}\right), \quad \mathrm{A}_{0}-\sqrt{\frac{\gamma-\kappa a^{2}}{2}}+A x+B y=0, \\
a\left(\gamma+\kappa a^{2}\right)<0 \Longrightarrow y=\ln \left(\sqrt{\frac{2}{r-\kappa a^{2}}} \frac{1}{t+\mathrm{A}}\right), \quad \mathrm{A}_{0}+\sqrt{\frac{\gamma-\kappa a^{2}}{2}}+A x+B y=0,
\end{array}
$$

with $\mathcal{\kappa}$ as in (3.32).

\section{Discussion and Conclusion}

In this work we have taken advantage of transformations provided for the Abel equations of the first kind. Equations of this kind are obtained by a proper general transformation and they represent the reduced forms of two general subclasses of nonlinear autonomous equations of the second order investigated here. Further, in a previous work we have considered a specific Abel equation including arbitrary functions, which can be solved in closed-form, and using another transformation (introduced by Kamke), we have finally derived a sufficient condition yielding an implicit solution of a general (Ab-1) equation. We note in particular that the arbitrary functions are finally eliminated, "allowing" the derivation of this closedform solution.

Moreover in this work, another arbitrary function, introduced in the analysis, takes advantage of the extracted solution (for an (Ab-1) equation), yielding exact one-parameter closed-form solutions concerning the original autonomous equations. Hence we can assert that, although arbitrary, all these functions have been profitably used in the analysis developed in these works.

In conclusion, regarding the choice of appropriate analytical tools we can claim that the use of Abel equations of the first kind gains an advantage over other analytical methods as far as certain general classes of autonomous nonlinear second-order ODEs, as well as equivalent to these forms two-dimensional autonomous nonlinear systems of first-order ODEs, are concerned.

\section{References}

[1] G. Duffing, "Erzwungene Schwingungen bei Veränderlicher Eigenfrequenz,” Braunschweig, vol. 134, 1918.

[2] B. van der Pol, “On relaxation oscillations,” Philosophical Magazine, vol. 2, no. 7, pp. 978-992, 1926.

[3] L. Rayleigh, "On maintained vibrations," Philosophical Magazine, vol. 15, no. 1, article 229, 1883, see the "Theory of Sound", Dover, New York, NY, USA, 1945.

[4] S. Lynch, "Small amplitude limit cycles of the generalized mixed Rayleigh-Lienard oscillator," Journal of Sound and Vibration, vol. 178, no. 5, pp. 615-620, 1994.

[5] A. Lins, W. de Melo, and C.C. Pugh, On Liénard's Equation, vol. 597 of Lecture Notes in Mathematics, 1977.

[6] F. Dumortier and C. Li, "On the uniqueness of limit cycles surrounding one or more singularities for Liénard equations," Nonlinearity, vol. 9, no. 6, pp. 1489-1500, 1996. 
[7] R. E. Mickens, "Liénard systems, limit cycles, melnikov theory, and the method of slowly varying amplitude and phase," Journal of Sound and Vibration, vol. 217, no. 4, pp. 790-793, 1998.

[8] S. Lynch, "Limit cycles of generalized Liénard equations," Applied Mathematics Letters, vol. 8, no. 6, pp. 15-17, 1995.

[9] S. Lynch, "Generalized cubic Lienard equations," Applied Mathematics Letters, vol. 12, no. 2, pp. 1-6, 1999.

[10] J. Garcia-Margallo and J. D. Bejarano, "The limit cycles of the generalized Rayleigh-Liénard oscillator," Journal of Sound and Vibration, vol. 156, no. 2, pp. 283-301, 1992.

[11] S. Lynch and C. J. Christopher, "Limit cycles in highly non-linear differential equations," Journal of Sound and Vibration, vol. 224, no. 3, pp. 505-517, 1999.

[12] R. E. Kooij and C. J. Christopher, "Algebraic invariant curves and the integrability of polynomial systems," Applied Mathematics Letters, vol. 6, no. 4, pp. 51-53, 1993.

[13] H. H. Denman, "Approximate invariants and lagrangians for autonomous, weakly non-linear systems," International Journal of Non-Linear Mechanics, vol. 29, no. 3, pp. 409-419, 1994.

[14] W. T. van Horssen, "Perturbation method based on integrating factors," SIAM Journal on Applied Mathematics, vol. 59, no. 4, pp. 1427-1443, 1999.

[15] F. Verhulst, Nonlinear Differential Equations and Dynamical Systems, Springer, Berlin, Germany, 2nd edition, 1996.

[16] E. K. Ifantis, "Analytic solutions for nonlinear differential equations," Journal of Mathematical Analysis and Applications, vol. 124, no. 2, pp. 339-380, 1987.

[17] E. N. Petropoulou and P. D. Siafarikas, "Analytic solutions of some non-linear ordinary differential equations," Dynamic Systems and Applications, vol. 13, no. 2, pp. 283-316, 2004.

[18] I. Langmuir and K. B. Blodgett, "Currents limited by space charge between coaxial cylinders," Physical Review, vol. 22, no. 4, pp. 347-356, 1923.

[19] H. T. Davis, Introduction to Nonlinear Differential and Integral Equations, Dover, New York, NY, USA, 1962.

[20] A. D. Polyanin and V. F. Zaitsev, Handbook of Exact Solutions for Ordinary Differential Equations, Chapman \& Hall/CRC, Boca Raton, Fla, USA, 2nd edition, 2003.

[21] M. P. Markakis, "Closed-form solutions of certain Abel equations of the first kind," Applied Mathematics Letters, vol. 22, no. 9, pp. 1401-1405, 2009.

[22] E. Kamke, Losungmethoden und Losungen, B.G. Teubner, Stuttgart, Germany, 1983. 


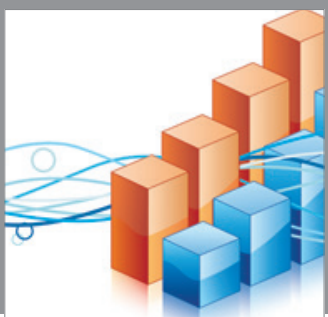

Advances in

Operations Research

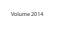

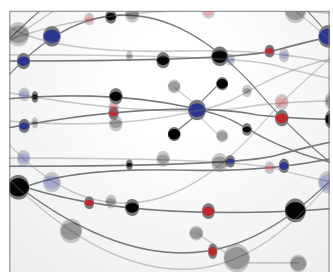

\section{The Scientific} World Journal
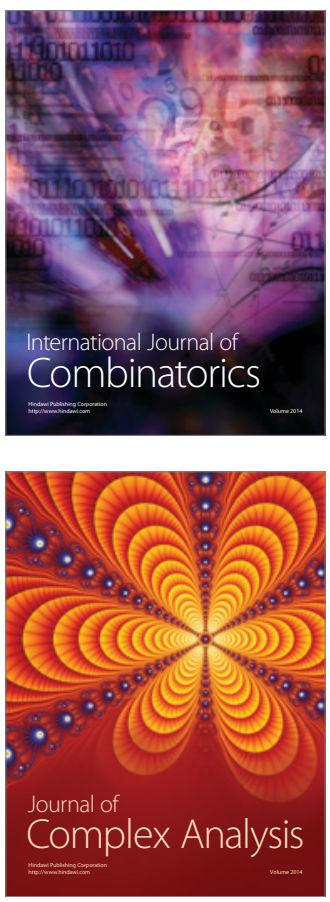

International Journal of

Mathematics and

Mathematical

Sciences
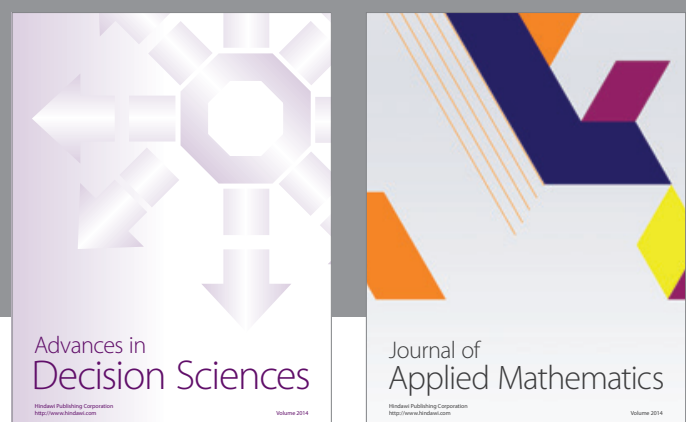

Journal of

Applied Mathematics
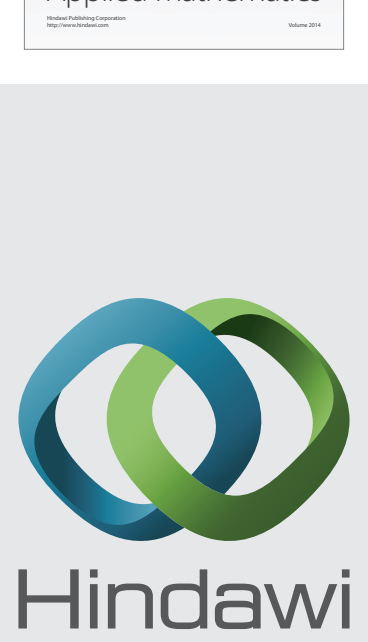

Submit your manuscripts at http://www.hindawi.com
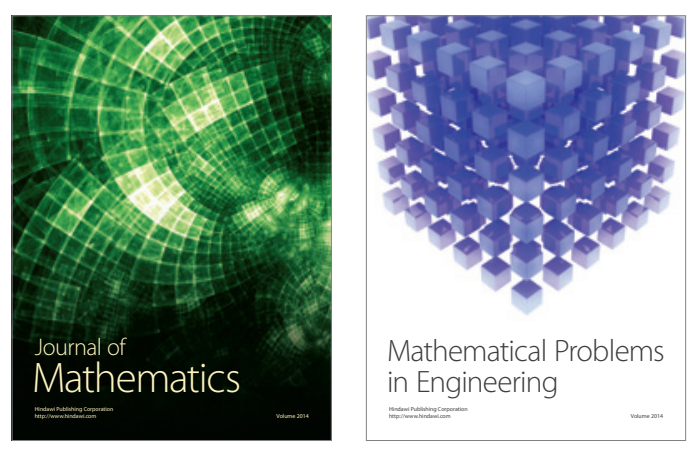

Mathematical Problems in Engineering
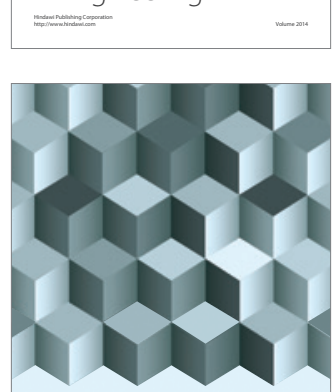

Journal of

Function Spaces
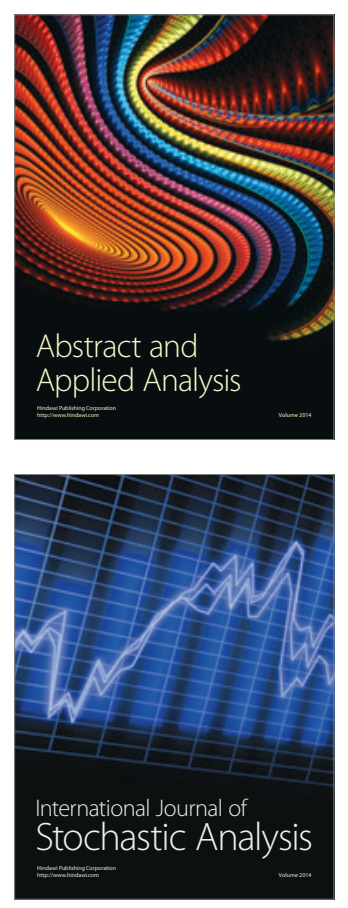

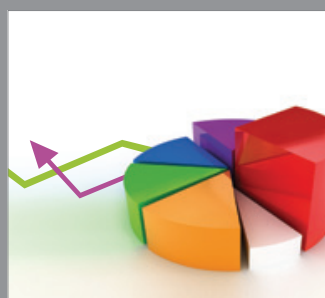

ournal of

Probability and Statistics

Promensencen
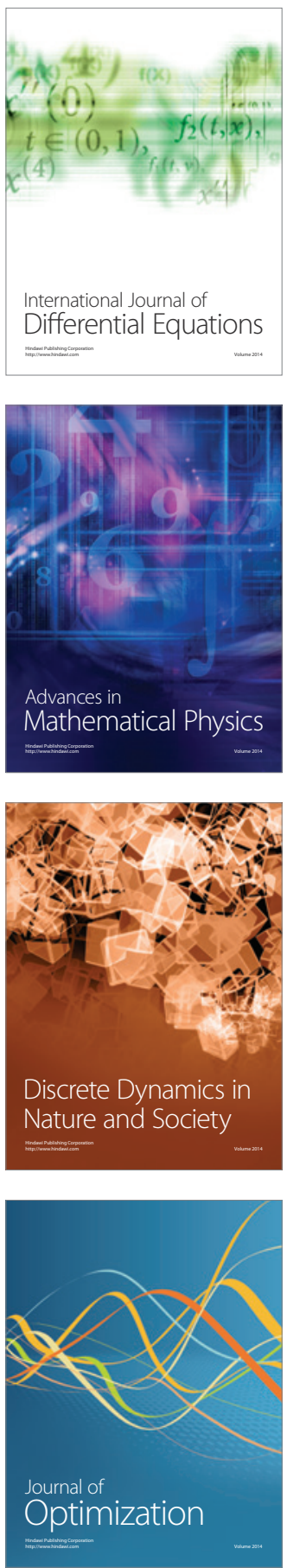